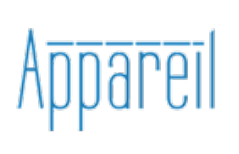

Appareil

23 | 2021

Poïétique du jeu vidéo

\title{
Ouvrir l'espace poétique potentiel des jeux vidéo
}

\section{Guofan Xiong et Chu-Yin Chen}

\section{(2) OpenEdition}

\section{Journals}

\section{Édition électronique}

URL : http://journals.openedition.org/appareil/4106

DOI : 10.4000/appareil.4106

ISSN : 2101-0714

Éditeur

MSH Paris Nord

Référence électronique

Guofan Xiong et Chu-Yin Chen, « Ouvrir l'espace poétique potentiel des jeux vidéo », Appareil [En ligne], 23 | 2021, mis en ligne le 31 mars 2021, consulté le 02 avril 2021. URL : http://

journals.openedition.org/appareil/4106 ; DOI : https://doi.org/10.4000/appareil.4106

Ce document a été généré automatiquement le 2 avril 2021.

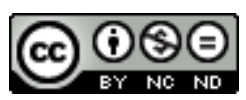

Appareil est mis à disposition selon les termes de la Licence Creative Commons Attribution - Pas d'Utilisation Commerciale - Pas de Modification 4.0 International. 


\title{
Ouvrir l'espace poétique potentiel des jeux vidéo
}

\author{
Guofan Xiong et Chu-Yin Chen
}

\section{Introduction}

Depuis plusieurs années, l'industrie du jeu vidéo s'essaie à différentes expériences poétiques, en utilisant surtout des ressources narratives, visuelles ou sonores... Pourtant, il est assez difficile de trouver un jeu vidéo dont le gameplay ${ }^{1}$ s'inspire $^{\prime}$ véritablement des formes de la poésie traditionnelle, alors même qu'elle possède nombre d'éléments jouables et ludiques.

1 Nous voudrions étudier la possibilité de trouver dans le gameplay du jeu vidéo le moyen d'accompagner, voire d'engendrer, une véritable poésie vidéoludique. Pour ce faire, nous commencerons par analyser les ressorts d'une certaine poétique du jeu, en étudiant les similitudes processuelles qu'offrent certains jeux et certaines traditions littéraires, puis nous évoquerons nos propres expériences artistiques qui, s'ancrant dans le champ du game art ${ }^{2}$, tentent de faire naître une authentique expérience poétique vidéoludique.

\section{Une approche de la poétique des jeux}

2 La poésie est une forme de littérature qui mobilise les qualités esthétiques et rythmiques du langage - telles que la phonesthésie, la symbolique du son et le mètre pour évoquer des significations en plus ou à la place du sens ostensible prosaïque. Dans la poésie traditionnelle asiatique, il y a toujours des règles formelles profondément liées à son environnement culturel d'origine. Ces règles peuvent porter sur des rimes, des limites de thèmes et de mots, des contraintes phonétiques... La manière dont les auteurs utilisent tous ces éléments pour créer une expérience artistique est une clé pour comprendre l'œuvre profondément enracinée dans cette culture. Nous voudrions 
prendre le pari ici qu'elles peuvent nous fournir une perspective intéressante sur la façon dont nous jouons dans différentes cultures.

Par exemple, pour créer un haïku traditionnel en japonais, il faut suivre deux règles générales. Tout d'abord, il doit se composer de trois petits vers qui ont cinq, sept et cinq mores ${ }^{3}$ (ou syllabes), puis il faut utiliser des mots qui présentent les saisons directement ou indirectement : «printemps » (春) ou « fleur de sakura » (さくら - 桜) sont, par exemple, deux mots saisonniers qui indiquent le printemps sur le calendrier lunaire. De plus, tous ces mots saisonniers présentent également un sentiment (souvent nostalgique) qui domine toute la poésie. À l'intérieur de ces contraintes, les poètes créent leurs expressions et leurs images personnelles sous forme littéraire. On peut considérer qu'écrire un haïku est comme jouer à un jeu de création : les groupes de mots japonais culturellement liés aux saisons (nature, traditions, fêtes, activités...) peuvent être vus comme des blocs de construction avec lesquels le poète joue. Le plaisir et défi du haïku ne consistent pas seulement à respecter la structure mais à créer la combinaison parfaite qui présente une image, une situation ou une sensation exprimant le sentiment exact du poète et éventuellement évoquant beaucoup plus dans l'esprit du lecteur.

Nous pouvons considérer que le poète qui suit des règles est comme le joueur qui affronte un gameplay. D'où notre question : pourrions-nous créer des jeux fondés sur les règles de ce type de poésie?

\section{Règles, poésie et jeu vidéo}

Figure 1. A Slow Year de lan Bogost
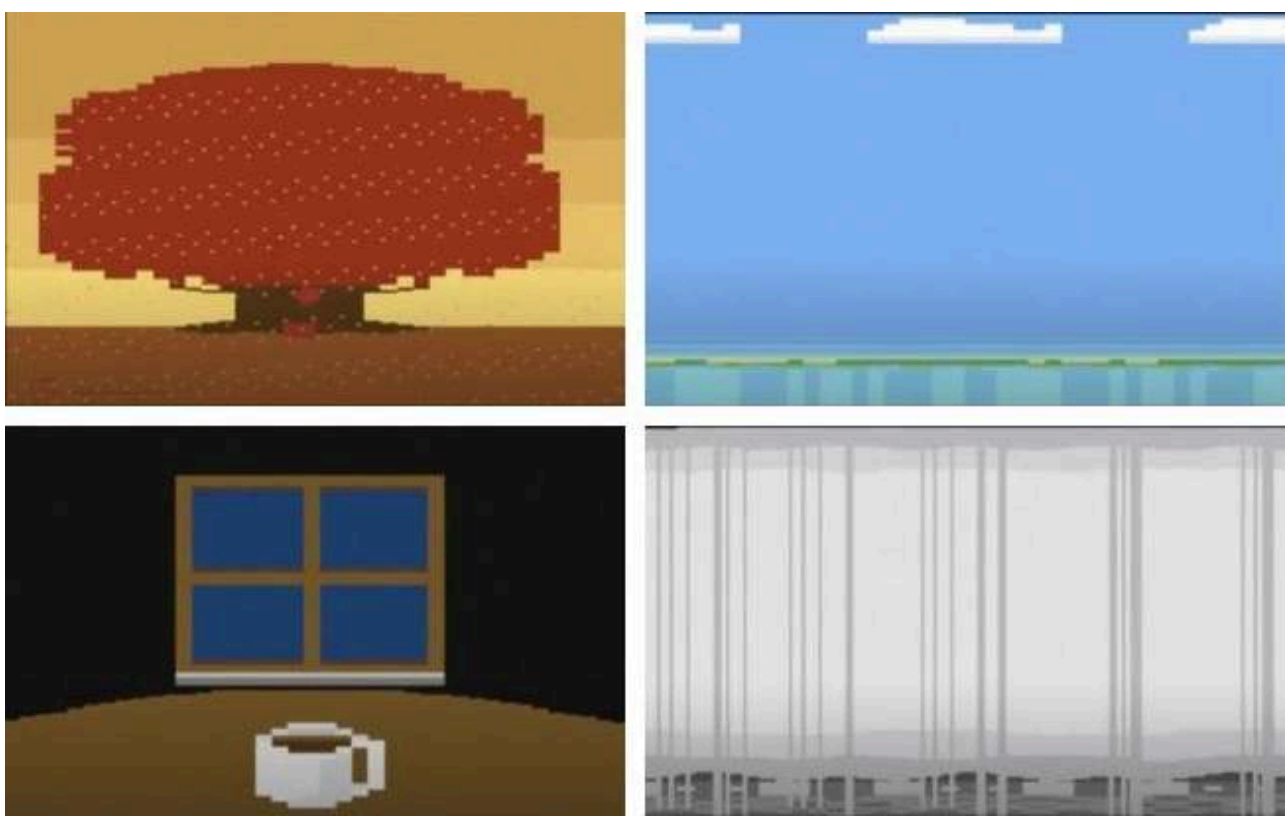

Source : Ian Bogost, A Slow Year, 2010 (crédits image : lan Bogost)

A Slow Year (Une année lente, fig. 1) est le game poem créé par Ian Bogost basé sur le haïku américain qu'il a lui-même écrit. A Slow Year est une collection de quatre jeux un pour chaque saison - fondée sur l'observation des choses. Ces jeux ne comportent ni de l'action ni de la stratégie : chacun d'eux nécessite des types différents d'observation 
calme et de contribution méthodique. C'est une œuvre très intéressante, qui présente les sensations et les expériences que le créateur a mises dans sa poésie. Comme le créateur du jeu l'a indiqué, le jeu tente d'embrasser une contrainte expressive maximale dans un condensé représentationnel: «En tant que jeux, ceux-ci reposent sur la représentation procédurale d'une idée que le joueur manipule. En tant que poésie, ils s'appuient sur la condensation de symboles et de concepts plutôt que sur la clarification d'expériences spécifiques. En tant qu'images, ils offrent des peintures visuellement évocatrices bien qu'obscures de scènes et d'objets réels ${ }^{4}$ ».

5 Le graphisme et les sons du jeu présentent parfaitement le sentiment nostalgique de «l'époque Atari ${ }^{5}$ ». Pour le créateur, les éléments symboliques utilisés autrefois en raison de la limitation des technologies sont également une partie poétique du projet lui-même, tout comme nous créons des éléments symboliques dans la poésie par les mots. Néanmoins, pour les joueurs d'autres générations ou non-joueurs du jeu vidéo, tous ces éléments perdent leur sens. Cela signifie que, pour comprendre le jeu, le joueur doit au moins lire la poésie écrite dans le livre et avoir une connaissance de base du média vidéoludique.

6 Le projet ne nous semble donc pas entièrement satisfaisant. Après y avoir uniquement joué, le joueur ne comprendra pas ce que sont les éléments poétiques à l'intérieur du jeu, pas plus que les élégantes compositions du haïku que le créateur a voulu présenter. Nous pouvons également déplorer le fait que tous les éléments ludiques de la poésie soient ignorés dans ce projet. Dans la plupart des jeux poétiques, la totalité du gameplay est utilisée pour renforcer une expérience poétique fondée sur un poème écrit par le créateur. Les joueurs n'ont pas accès aux formes et aux règles originales de la poésie lorsqu'ils jouent. Nous pouvons alors considérer que l'artiste créateur utilise le système de jeu pour écrire une poésie intégrant les éléments et les règles des supports de jeux vidéo, mais le plaisir de créer de la poésie avec ses formes originales reste absent pour le joueur. Un moyen d'y parvenir serait d'implémenter au cœur d'un jeu vidéo les règles de poésie qui sont celles du créateur. Or, il nous semble qu'un jeu fonctionne déjà selon ce principe : le mah-jong.

\section{Règles ludiques et poétiques : analogie entre le mah-jong et le juéjù}

7 Le mah-jong est un jeu de société créé et formé en Chine au début de la dynastie Qing (xvIII siècle). Il se joue à quatre joueurs avec un nombre de tuiles variable, généralement 144. Dans la plupart des variantes, chaque joueur commence par recevoir treize tuiles. À leur tour, les joueurs tirent et défaussent des tuiles, jusqu'à ce qu'ils complètent une main gagnante en utilisant la quatorzième tuile tirée pour former quatre combinaisons et une paire. Chaque joueur représente un vent soufflant dans l'une des quatre directions, en commençant par l'est, passant par le sud et l'ouest et terminant par le nord. Dans la culture chinoise ancienne, les quatre directions renvoient à l'idée de la Terre : la forme carrée présente la construction du monde humain (fig. 2). 
Figure 2. Forme carrée du mah-jong (gauche) ; représentation du monde dans une forme carrée, sur un miroir en bronze (droite)
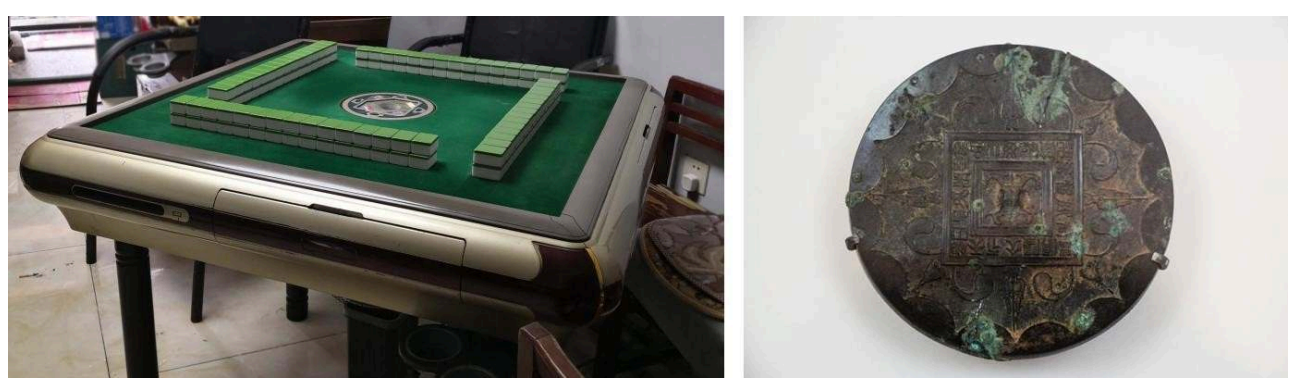

Source : Table de mah-jong (crédits image : Qifeng Ren, 2020), Miroir en bronze, I siècle, MA1093, Musée national des arts asiatiques - Guimet (MNAAG) (crédits image : Guofan Xiong, 2020)

Il est notable qu'une forme poétique chinoise, le juéjù (绝句), utilise ce même concept de forme carrée. En effet, ce dernier comporte toujours quatre vers - c'est un quatrain - et, en outre, les phrases sont généralement utilisées pour décrire la nature ou l'environnement créé par l'homme qui reflète les émotions et les idées du poète. Par exemple, dans le poème "Neige sur le fleuve ${ }^{6}$ », les deux premières phrases décrivent l'environnement, les montagnes éloignées et la route enneigée, les deux dernières les éléments les plus proches : le vieux pêcheur et la rivière gelée. Chaque phrase apporte un élément à l'image entière et elles présentent toutes un sentiment de solitude. Tout comme les quatre joueurs du mah-jong qui complètent le cercle du jeu, les phrases complètent le monde blanc et froid à l'intérieur de cette poésie.

\section{江雪}

千山鸟飞绝

万径人踪灭

孤舟蒿笠翁

独钓寒江雪

Neige sur le fleuve

Sur mille montagnes, aucun vol d'oiseau.

Sur dix mille sentiers, nulle trace d'homme.

Sur une barque solitaire, un vieillard en manteau, chapeau,

Pêche seul la neige sur un fleuve de glace ${ }^{7}$.

柳宗元 (Liŭ Zōngyuán)

Or, l'objectif des joueurs de mah-jong est justement de composer avant les autres une forme spécifique, forme similaire à celle d'une phrase de juéjù qui utilise cinq caractères (fig. 3). Pour gagner un tour, vous devez avoir cinq sets : quatre premières combinaisons composées de trois éléments (peut-être un chow, un pung ou un kong ${ }^{8}$ ) et une paire. Nous pouvons donc considérer que les quatre premiers ensembles présentent les quatre caractères d'une phrase, tandis que la paire, en tant que dernier caractère, fonctionne comme la rime reliant les phrases entre elles. Ce premier rapprochement se complète par la façon dont se détermine la réussite du joueur : le jeu ne se gagne pas à tour de rôle, mais en calculant des crédits. 
Figure 3. Forme similaire entre le mah-jong et la phrase de juéjù
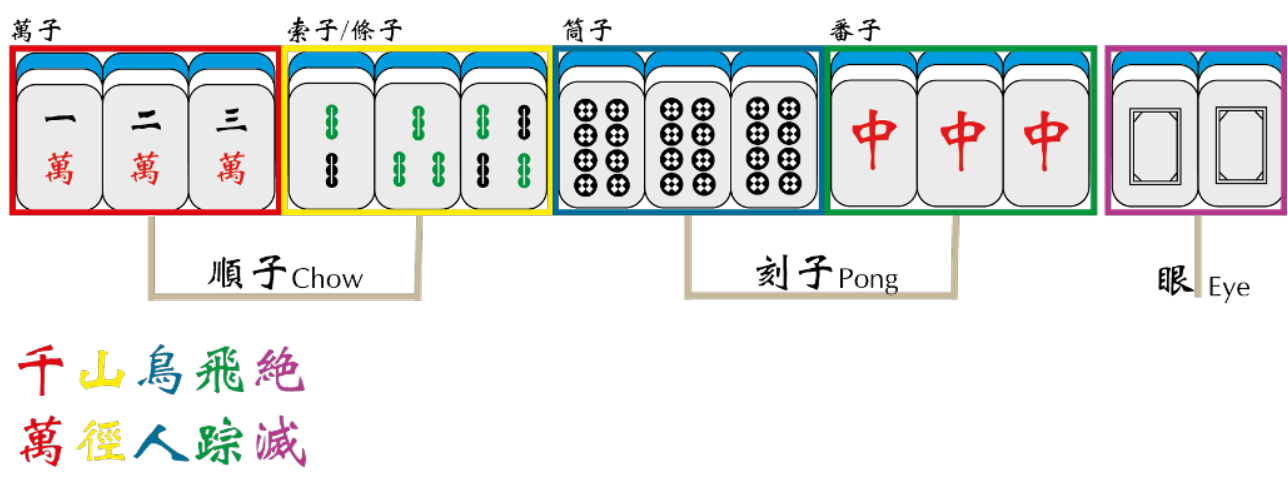

Source : Guofan Xiong, 2021

Lorsque la première personne déclare avoir réussi à composer une forme, il peut choisir d'arrêter le tour. Ensuite, les autres joueurs vérifient combien la forme qu'il a composée vaut de points et ils perdent les points correspondants au profit du joueur gagnant. La valeur des points dépend de la forme de base et du nombre de combinaisons spéciales que les joueurs ont composées en collectant les 144 tuiles. Une victoire de base soutire un point à chaque joueur perdant, mais les formes les plus élevées peuvent accaparer 88 points chacune.

Ces formes ont souvent des significations spécifiques tirées de la culture chinoise. Par exemple, si un joueur a collecté trois sets qui contiennent des numéros de la même couleur de un à neuf, un autre set et une paire, il soutirera seize points aux autres joueurs, car il a non seulement composé la forme de base, mais également une grande suite (un dragon). Autre exemple, lorsqu'un joueur a collecté les combinaisons des tuiles qui indiquent les vents des différentes directions, il peut gagner le maximum soit 88 points des autres joueurs, car les vents représentent les quatre saisons et le monde (fig. 4). Tout comme écrire un juéjù ne consiste pas seulement à remplir vainement une forme définie par des règles, la réussite au mah-jong nécessite de composer des formes significatives qui valent un plus gros score. Au mah-jong, les formes de base donnent peu de gains au joueur débutant, là où des compositions plus osées et un jeu plus hardi rapportent rapidement plus de points; dans le juéjù, les tournures audacieuses et les exceptions ont toujours plus de valeur que les productions faites par la seule application des règles de base. Si un joueur de mah-jong a composé sept doubles, il obtiendra les 88 points complets de chaque joueur; or, certains des plus beaux poèmes chinois ont sept doubles mots...

\section{声声慢}

\section{寻寻受受,}

冷冷清清,

凄凄惨惨戚戚。

乍喛还寒时候, 最难将息?...

\section{Shēngshēngmàn}

Agité et perdu,

Froid et solitaire,

Infortuné, misérable et anxieux. 
Chaleur précoce entrecoupée de froid, moment le plus difficile pour la convalescence $^{10} \ldots$

李清照 (Ľ̌ Qīngzhào)

Figure 4. Combinaison dragon (gauche); combinaison quatre saisons (droite)
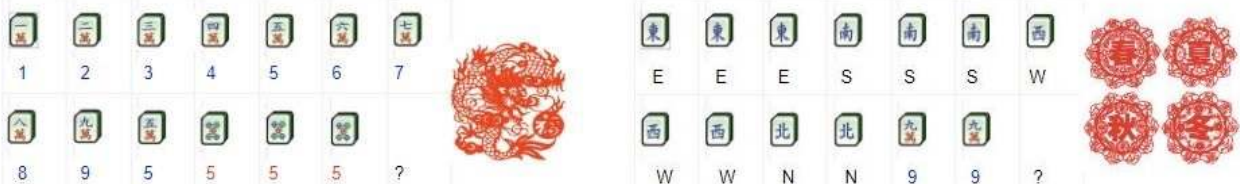

Source : Guofan Xiong, 2017 ancienne. Ces formes sont des représentations du fonctionnement de notre monde et de ce qui les rend spéciales dans cette culture même. Les juéjù ont été initialement utilisés pour créer des situations où le lecteur peut ressentir à la fois l'émotion et l'esthétique captées par le poète, tandis que le mah-jong, un jeu de hasard axé sur la compétition, ne cherche pas à donner un sens à son espace de jeu. Y a-t-il alors une activité hybride entre la littérature et le gameplay qui peut nous aider à chercher les raisons pour lesquelles les règles du mah-jong ne supportent pas une expérience poétique? Que devons-nous faire pour créer cette-dernière lors de la création d'un jeu inspiré de la poésie traditionnelle?

\section{Jeu littéraire et gameplay}

Le duìduizi (对对子) est une autre pratique pouvant révéler un aspect des liens entre poésie et jeu. Il s'agit d'un jeu littéraire joué par des érudits à travers l'histoire de la Chine. Normalement joué par deux joueurs, le premier compose une phrase qui suit les règles et les rythmes exacts du juéjù, puis l'autre doit composer une seconde phrase correspondant parfaitement à la phrase du premier. Les deux phrases doivent former un couplet et refléter à la fois leur forme et leur signification. Par exemple, si le premier caractère de la première phrase est dà (大) l'adjectif "gros ", le premier caractère de la deuxième phrase doit être un autre adjectif dont la signification est liée à la taille (par exemple : petit, grand, long...). Ensuite, le deuxième joueur compose une autre phrase pour que le premier joueur termine le couplet, formant un duilián ${ }^{11}$ (对联). Le jeu s'arrête lorsque l'un des érudits échoue à terminer ou créer une phrase correcte avec un sens intéressant. Comme dans le mah-jong, les joueurs tentent de compléter une forme spécifique en échangeant leurs composants et la réussite ne se limite pas à remplir des formes, mais bien à composer une forme qui ait du sens. Les différences entre les deux jeux sont que le duiduizi crée toujours de nouveaux distiques alors que les formes possibles en mah-jong sont limitées. Le duiduizi est souvent joué dans un espace public ou formel, le prix pour le vainqueur étant la dignité, qui est considérée comme la fortune la plus importante pour un ancien universitaire chinois. Quant au perdant, il perd la face et sort humilié. 
Figure 5. Oh...Sir!! The Insult Simulator

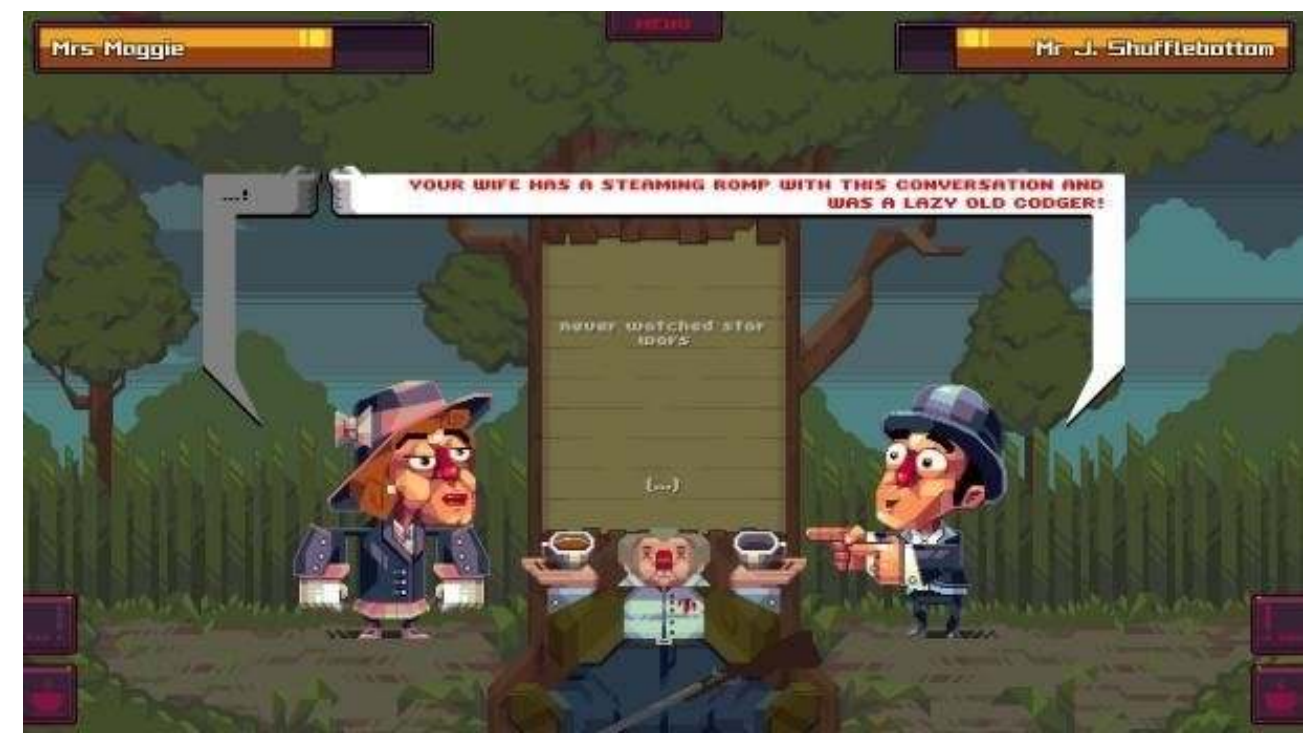

Source : Oh...Sir !! The Insult Simulator, Vile Monarch, 2016 (crédits image : Guofan Xiong, 2020)

Dans le jeu Oh...Sir!! The Insult Simulator (2016), une certaine partie du gameplay ressemble aux règles du duiduizi (fig. 5). Comme les créateurs l'indiquent, l'idée originale est de créer un jeu de combat où vous utilisez des mots pour nuire à la fierté de votre adversaire. Les joueurs choisissent chacun un avatar (chaque avatar a ses faiblesses pour certains types d'insultes) qui représente un stéréotype de la culture anglaise. Ils composent à tour de rôle une phrase en choisissant des mots donnés au hasard, dans un laps de temps donné. Les mots sont les armes du simulateur d'insulte, la structure de la phrase est fondée sur la grammaire anglaise et les meilleurs scores s'obtiennent en composant une phrase correcte ayant un effet maximum sur l'adversaire, autrement dit le plus bel effet esthétique.

Au mah-jong, toutes les formes ont été définies dans les règles et tous les joueurs les connaissent avant de jouer. Dans le jeu Oh...Sir!!, bien que le simulateur donne une idée abstraite de la façon dont il valorisera la phrase insultante, les joueurs voient les dommages infligés à l'adversaire mais ne savent pas selon quelle règle ces dommages sont calculés par l'ordinateur et cette règle restera cachée. Le joueur peut en avoir une idée approximative, mais ne la comprendra jamais pleinement sans lire les codes préprogrammés par les développeurs. Il en va de même pour le duel de duiduizi en public, car le résultat est parfois basé sur la réaction du public alentour et même la confiance du lettré. Comme le mah-jong, l'Insult Simulator a un gameplay inspiré de la pratique de la littérature. Ces deux jeux très différents nous ont montré que des règles venant de diverses formes de littérature être adaptées en jeux et que nous pouvons créer de nouveaux types de gameplay à partir d'elles.

En jouant au mah-jong, on peut ainsi comprendre la forme d'une certaine poésie chinoise, voire le terrain culturel sous-jacent, sans même avoir une compréhension de la langue; là où des traductions ne donneront accès qu'aux significations et aux idées de cette poésie. Les jeux se révèlent ainsi propices à mettre les personnes dans une situation leur permettant d'explorer et d'apprendre de leur interaction avec les contraintes à l'intérieur de l'espace de jeu. Issus du même environnement culturel, les jeux traditionnels peuvent être une grande source d'inspiration lorsque l'on veut 
présenter une forme littéraire avec une approche ludique. Ils permettent d'étudier la façon dont nous pouvons représenter et sublimer les expériences poétiques, qui sont essentielles pour une forme littéraire comme la poésie. Dans le cas du mah-jong, pourtant, qu'est-ce qui empêche les joueurs de ressentir le sens poétique et les formes poétiques du jeu? À l'inverse, comment le duiduizi peut-il véhiculer un sens poétique? Dans Le Rêve dans le pavillon rouge $e^{12}$ (红楼梦), l'écrivain Cáo Xuĕqín (曹雪芹) a écrit une scène qui reflète la partie poétique du duiduìzi : après le dîner de famille de la fête de la lune, deux filles nobles vont dans un pavillon du jardin. Elles pensent toutes les deux à leur vie dans cette famille d'accueil extrêmement riche. Elles commencent à jouer au duiduìi. L'une des filles jette un rocher dans l'étang de lotus, puis une grue s'envole à cause du bruit. Inspirée par l'instant, elle compose sa phrase : « La grue jette une ombre sur l'étang glacial ${ }^{13}$ " (寒塘渡鹤影). Fière de sa création, elle attend que son amie compose sa pièce. La deuxième fille regarde la pleine lune dans le ciel, tandis qu'un pétale de fleur tombe d'un arbre à côté du pavillon. Elle répond lentement : «L'âme des fleurs enterrée dans le clair de lune froid ${ }^{14}$ » (冷月葬花魂). Dans ce roman, la scène est utilisée pour aborder l'état sentimental et le sort de ces deux filles nobles. Le duiduizi est le support expressif et poétique de leurs sentiments et de leur destin.

Ce que le duiduizi nous indique ici sont justement les éléments qui manquent au mahjong pour soutenir une expérience poétique. Par comparaison avec le mah-jong, le duiduizi a un rythme de jeu relativement flexible, les joueurs créent leurs expressions en composant des mots et la compétition est un motif simple qui invite les joueurs à chercher dans leurs connaissances et leurs sentiments. Le duiduizi permet à ses joueurs de capturer l'instant émotionnel qui a inspiré leurs expressions poétiques. Enfin, le duiduìzi place les joueurs dans l'univers qui leur permet d'apprendre l'aspect culturel des règles, plus encore: qui les invite à créer leurs expériences uniques. Dans ces domaines, le support de jeu vidéo pourrait fournir le même type de solutions aux problèmes des jeux traditionnels.

\section{La poésie des jeux vidéo}

\section{Le rythme}

18 Les joueurs décrivent généralement leur expérience positive d'un jeu avec des mots comme " curiosité », « excitation », « défi », « exaltation » et «triomphe $»^{15}$. La plupart de ces mots sont reliés au défi et à leurs capacités. L'expérience optimale ${ }^{16}$ culmine dans le Flow, définit par le psychologue Mihály Csíkszentmihályi comme suit: «Être complètement impliqué dans une activité pour elle-même. Oublier son ego. Le temps passe. Chaque action, mouvement et pensée découlent inévitablement de la précédente, comme pour le joueur de jazz. Tout votre être est impliqué et vous utilisez vos capacités au maximum ${ }^{17}$.»

19 Cette expérience se substitue à la réalité car le joueur est complètement happé par le temps et les situations du jeu. D'un point de vue commercial, le jeu vidéo est peut-être l'instrument parfait pour apporter une expérience de Flow. Dans le domaine du développement de jeu vidéo, un designer de haut niveau est quelqu'un qui sait concevoir les niveaux de difficulté et la dynamique de jeu, pour que le joueur vive une expérience optimale et joue le plus longtemps possible. 
Le mah-jong est un jeu relativement intense, qui requiert à la fois de la chance et des capacités : les joueurs entreront facilement dans un état de Flow. Dans la conception de jeux vidéo, certains développeurs soutiennent que cette notion de plaisir peut les aider à créer un gameplay plus émotionnel ${ }^{18}$.

Ainsi, le jeu vidéo Journey de Jenova Chen a été largement apprécié par les joueurs et les critiques. Pour ces derniers, l'expérience du jeu peut être considérée comme une expérience esthétique et le jeu comme une œuvre d'art. Les adjectifs "beau», " émotif », "sensible » et "poétique » employés souvent pour décrire une poésie, peuvent parfaitement évoquer les sensations ressenties après avoir joué à Journey.

Pendant ses études supérieures, le directeur créatif de Thatgamecompany, Jenova Chen, est devenu obsédé par le concept de Flow. Chen a consacré sa thèse de Master of Fine Arts à l'application du Flow dans les jeux.

Il pense que la théorie des flux fournit un modèle de travail aux concepteurs de jeux, les encourageant à garder les joueurs dans une zone idéale propice à l'expérience du Flow, entre l'anxiété et l'ennui (fig. 6). Toutefois, Journey fournit la preuve que l'équilibre entre le défi et la capacité n'est pas la solution pour créer une expérience poétique dans le jeu : il offre une ouverture aux nouveaux joueurs grâce à un gameplay ajustable et à des mondes uniques et saisissants créés pour que les joueurs puissent les explorer, tout comme nous avons souvent besoin de la liberté de pouvoir ajuster notre propre vitesse lorsque nous lisons de la poésie. Loin des questions de défi ou de capacité, il s'agit de créer un lien émotionnel avec les mots et les symboles de la poésie, ce que réussit le gameplay de Journey.

Figure 6. Flow de Jenova Chen

\section{Challenge}

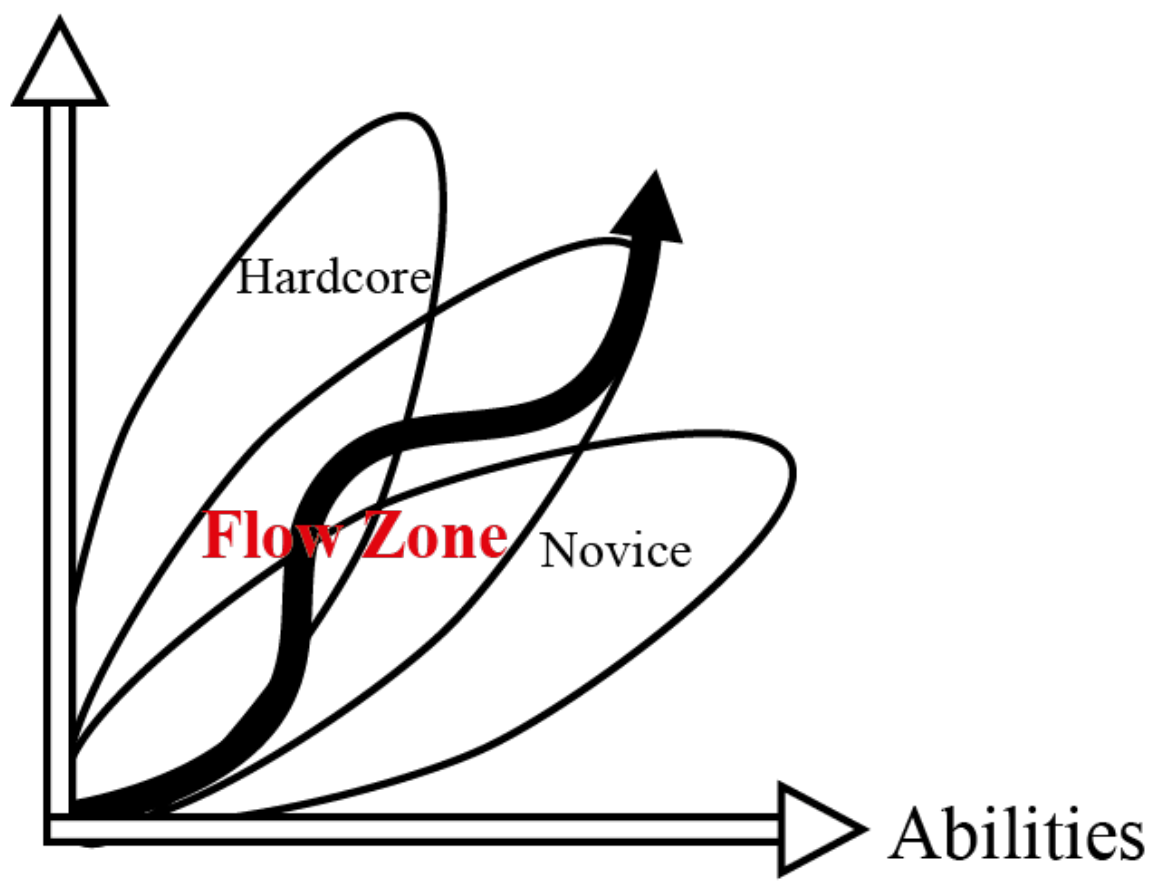

Source: Guofan Xiong, 2020, d'après Jenova Chen, «Flow in Games », mémoire de master, Université de Californie du Sud, 2006. 

dynamiquement en faveur de l'exploration simple, qui fait des joueurs des entités vivantes explorant l'environnement en détail dans un rythme détendu. Comme la plupart des jeux vidéo, Journey tend à offrir un espace continu, plutôt que discontinu, un espace qui doit être traversé délibérément et activement, à l'opposé de ce qui se passe avec la découverte d'un spectacle panoramique. Il est demandé aux joueurs d'interagir sur l'environnement, avec des inconnues qui surgissent tout le long du trajet. Le jeu efface le sens de l'arrêt ou de l'étape et le but du voyage, en renforçant l'expérience poétique du voyage lui-même.

Le designer de Journey a voulu restaurer le plaisir du temps, de la durée du voyage, perdu par l'accélération de la vie moderne. Ainsi, ce jeu prétend offrir des rêves aux jeunes vivant en ville. Il n'explique rien et ne s'en excuse pas. Si le voyage de Journey est celui de n'importe qui, alors il peut signifier tout ce que nous voulons. À travers le jeu, Jenova Chen et Kellee Santiago (les concepteurs du voyage), créent un espoir d'exploration et d'autres émotions positives chez les joueurs; émotions qu'ils ne ressentent pas avec d'autres sortes de jeux ${ }^{19}$. En d'autres termes, le gameplay est ici conçu comme un mécanisme devant aider les joueurs à se découvrir eux-mêmes ainsi que leurs émotions, plutôt que de se concentrer sur les tâches à accomplir ou sur les actions de leurs adversaires. Il rappelle ainsi aux joueurs l'existence du temps dans les situations de leur quotidien plutôt que de les en sortir.

\section{L'instant}

Le juéjù a un lien profond avec le lavis chinois. Ces deux formes littéraire et artistique se présentent souvent ensemble pour former une expression poétique complète.

Texte et image, elles sont comme le Yin et le Yang. Elles ont les mêmes origines et sont interconnectées, mais en même temps, elles sont différentes par la nature de leur support. La poésie est l'art du temps quand la peinture est l'art de l'espace ${ }^{20}$.

L'image poétique sera alors celle qui condense un "esprit du temps » dans un lieu spatial, cette temporalité pouvant prendre deux formes principales : celle de la vignette instantanée et celle d'un univers atemporel. Par exemple, il existe plusieurs anciens lavis chinois qui offrent une expérience picturale du poème « Neige sur le fleuve ». Les deux plus célèbres partagent le même nom : Pêcheur à la ligne solitaire au bord d'une rivière en hiver (寒江独钓图) ${ }^{21}$.

L'artiste Mă Yuăn ${ }^{22}$ (马远) a présenté l'image délicate d'un vieil homme pêchant dans son bateau tel que mentionné dans la poésie, tandis que le reste de l'environnement est laissé vide. Il ne représente pas tous les éléments décrits dans la poésie, au contraire : il les efface pour se concentrer sur le renforcement du sentiment froid et solitaire dominant la poésie originelle. Des centaines d'années plus tard, un autre artiste, Zhū Ruì ${ }^{23}$ (朱瑞), a peint sa propre version. Dans celle-ci, les phrases de la poésie sont représentées par des visuels très détaillés. L'intention ici est claire : l'artiste veut créer un espace émotionnel par les paysages, qui ont un lien plus fort avec le poème. Comme l'a indiqué le critique chinois Dá Chóngguāng ${ }^{24}$ (笪重光) : « dans un lavis chinois, le vide se mélange avec la présence, il transforme la présence en vide, l'environnement en émotion. L'espace vide peut susciter plus d'inspiration et d'imagination ${ }^{25} »$. Suivant ce concept, tout comme les vignettes, les lavis créent souvent des émotions chez le 
spectateur. Les couleurs, les espaces, les compositions et les autres éléments symboliques dans les lavis sont tous devenus des outils pour renforcer cette expérience.

En littérature, une vignette est une description ou un compte rendu bref, indéfini et évocateur d'une personne ou d'une situation. Selon Bogost, les vignettes sont généralement destinées à donner un sens à un personnage, plutôt qu'à faire avancer un récit. Comme dans un croquis, les vignettes sont impressionnistes et poétiques, représentant une expérience ou un environnement ${ }^{26}$. Or, certains jeux vidéo, brefs, sans narration spécifique, imitent la vignette.

L'œuvre Passage de Jason Rohrer (2007) utilise des techniques de brouillage pour changer la façon dont les jeux vidéo présentent la mort (fig. 7). La mort s'évoque, mélancolique, comme dans un memento mori. Peu importe ce que les joueurs choisissent de faire dans le jeu, ils mourront de toute façon dans un délai de cinq minutes. Le joueur commence sur le côté gauche, sous l'apparence d'un homme pixélisé, blond aux yeux bleus. Son avatar se déplace lentement vers la droite de l'écran, tandis que les cheveux grisonnent et que le corps ralentit, jusqu'à ce qu'il finisse par mourir. Rohrer (2008) note que le «score semble assez dérisoire au-dessus de l'image de votre petite pierre tombale ${ }^{27}$ \#. Cela peut frustrer les joueurs qui veulent que le jeu réponde à leurs attentes d'un monde hérö̈que. Jouer à Passage est comme lire une phrase, de gauche à droite, du début à la fin.

Un détracteur de Passage, Harry Giles, prétend que c'est « un exercice pour mettre à nu les structures sémiotiques les plus simples du voyage à travers la vie. Par conséquent, il ne peut jamais nous dire tout ce que nous ne savons pas encore ${ }^{28}$ ». L'œuvre ne raconte pas une histoire pour faire sortir le joueur de sa réalité quotidienne, mais pour lui faire prêter attention au moment qu'il passe dans le jeu et lui faire ressentir une impression de vide. Comme le dit Rohrer, le jeu semble demander au joueur : maintenant que vous vous souvenez de votre propre mortalité, qu'allez-vous faire du reste de votre vie ${ }^{29}$ ? Le jeu Passage pose une question personnelle et philosophique. Tout comme les lavis de Mă Yuăn, Passage utilise son approche artistique et son design minimal pour générer un impact émotionnel sur ses joueurs qui peut aller de la mélancolie aux regrets aigresdoux, en passant par l'ennui ${ }^{30}$.

\section{L'univers intemporel}

La vignette immerge le lecteur dans une situation très spéciale, qui lui fait ressentir l'émotion transmise par la poésie : c'est l'instant capturé par le poète. Par ailleurs, elle engendre également un environnement issu de l'espace décrit, un univers qui regroupe tous les éléments visuels et symboliques portés par l'expérience poétique.

Appelons « espace de jeu » l'espace virtuel dans lequel se déroule un jeu vidéo. L'espace virtuel est le "cercle magique» ou le terrain de jeu du médium jeu vidéo. Le concepteur de ce dernier jeu crée une réalité dans laquelle l'individu joue le jeu. Ici, la réalité virtuelle se comprend comme un environnement créé par un ordinateur ou tout autre média et dans lequel l'utilisateur a le sentiment d'être présent ${ }^{31}$.

Dans un jeu vidéo, la création de l'espace de jeu est la partie la plus importante. La façon dont les créateurs utilisent ces éléments dans le monde du jeu définit l'expérience qu'ils veulent faire vivre au joueur. À la différence des autres médias culturels comme le cinéma ou le théâtre, le jeu vidéo ne crée pas le héros de l'histoire en premier. Au contraire, il crée d'abord le monde (règles, logiques, physiques...). Les 
histoires se déroulent à partir des interactions entre le héros (le joueur) et le monde ${ }^{32}$. Le jeu vidéo The Witness nous montre une manière intéressante d'utiliser tous ces éléments dans un univers de jeu, ce qui conduit à enrichir l'expérience d'un jeu traditionnel confinant à une discussion philosophique.

The Witness est un jeu de puzzle. Sur une île mystérieuse, presque toutes les surfaces libres ont un panneau avec un puzzle linéaire. Après avoir terminé un puzzle, les joueurs débloquent un autre espace doté d'un autre puzzle, jusqu'à achever une région. Après avoir terminé onze zones autour de l'île sans nom, le joueur se rendra au centre pour résoudre les énigmes de la zone finale.

Pour trouver les solutions des puzzles, il faut tenir compte de tout l'espace de jeu : les reflets dans l'eau, la rivière qui passe dans la vallée, les perspectives, les ouvertures des fenêtres sculptées, la connexion des branches, les ombres sur le puzzle, la tonalité des chants d'oiseaux... (fig. 8) Le jeu n'offre aucun contexte dans lequel placer ses connaissances. L'histoire, dans la mesure où il y en aurait une, est également difficile à comprendre. Il y a des vidéos et des extraits audio cachés sur l'île qui qui exposent un d'un cadre philosophique du monde. Tous ces mécanismes conduisent les joueurs à adopter un mode de jeu fondé sur la recherche des détails ${ }^{33}$. Parfois, les puzzles peuvent rester difficiles à résoudre pour les joueurs; ceux-ci s'arrêtent, puis ils trouvent des solutions en observant, en réfléchissant ou en cherchant à l'intérieur et à l'extérieur du jeu.

Figure 7. Solutions de puzzles de The Witness dans l'environnement
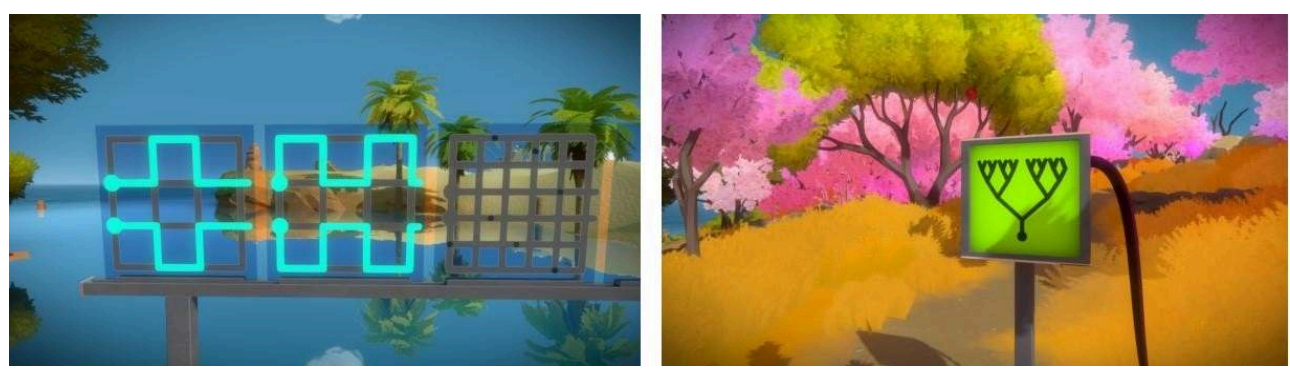

Source : Jonathan Blow, The Witness, 2016 (crédits images : Guofan Xiong, 2020)

Le puzzle est le gameplay, l'idée et le centre du jeu. Jouer à The Witness est comme jouer à un puzzle linéaire : le vide ressenti à la fin par le joueur ressemble à celui ressenti après avoir suivi un labyrinthe avec un crayon sur un papier. Ce qui est important, c'est la recherche de la sortie, non la sortie elle-même. The Witness présente parfaitement un argument de philosophie en utilisant son gameplay, son univers sonore et vidéo : il met en évidence la question de notre relation à la recherche de la vérité. Il s'agit de l'exploration du besoin humain de tendre la main et de comprendre l'inconnu. C'est la logique même derrière la raison pour laquelle nous jouons avec un puzzle : la vérité est moins importante que la méthode pour la rechercher.

Dans des jeux comme ceux-ci, les créateurs ne cherchent pas à donner une narration définie au jeu, mais à utiliser un gameplay caractérisé pour faire l'expérience d'une mathesis, exemplifier une recherche éternelle, labyrinthique et à jamais mystérieuse, car ne révélant jamais son objectif. Ces jeux en disent long sur le fonctionnement de l'expérience à l'intérieur de l'espace de jeu et sur la sensation que l'on éprouve dans une situation de jeu. Ils ne se concentrent pas seulement sur le fait de donner du sens à sa mécanique ; mais aussi sur la manière d'interagir avec les graphismes, le son, le texte 
ou même l'histoire. Tout comme le juéjù et le lavis chinois, l'expérience poétique vient d'un univers complet incluant à la fois le temps et l'espace.

En générant une expérience poétique ou tout autre expérience artistique similaire, un système de jeu vidéo pourrait permettre de susciter de l'empathie ou de la créativité chez le joueur. Les jeux vidéo pourraient non seulement se concentrer sur la simulation réaliste des expériences, mais ils pourraient également offrir des visions métaphoriques d'idées, être capables de représenter la vérité subjective et l'individualité de leur créateur, tout en capturant ses sensibilités et ses intentions. Les jeux vidéo devraient alors créer des systèmes et des mondes qui invitent les joueurs à l'introspection, à progresser dans la compréhension et les idées sous-jacentes au gameplay.

\section{Exploration de l'espace poétique du jeu vidéo : exemples de game art}

Dans cette partie, nous utiliserons les méthodes de game art pour explorer les capacités du système de jeu vidéo à générer des significations personnelles et poétiques pour le joueur. Cela se veut moins une adaptation de formes littéraires qu'une recréation, qui s'inspire à la fois des jeux et aussi des règles et formes poétiques pour soutenir une expérience poétique transculturelle. De plus, il est prévu d'ajouter d'autres aspects culturels (cérémonies, interactions sociales, histoires...) dans le gameplay.

Dans notre projet, l'œuvre de game art est à la fois une installation numérique et un jeu vidéo autonome. L'œuvre utilise le gameplay comme matériau d'expression inspiré du système basé sur des règles et ambitionne une dimension ludique et un objectif de jeu complet. Il s'agit d'une installation interactive invitant à jouer et à explorer. Les méthodes de game art nous offrent deux avantages :

1. Le Game art, libéré du cadre du jeu vidéo, permet de mélanger le jeu vidéo avec d'autres médiums artistiques, d'explorer et de créer différents types d'expressions.

2. Le Game art dispose de deux avantages par rapport au contexte du gameplay: la réaction directe du joueur, la présentation devant un public peu familier du jeu vidéo ou de l'art numérique ${ }^{34}$.

\section{L'inspiration des formes poétiques}

Fortement inspiré par des jeux vidéo indépendants comme Flower (Thatgamecompany, 2009) et The Night journey (Bill Viola et Game Innovation Lab, 2007-2018), le jeu Return: Flower Bird Butterfly and Fish (回: 花鸟 虫鱼) est l'une des premières expérimentations que nous avons réalisées sur des supports de jeu vidéo (fig. 9). L'idée est d'utiliser le système de jeu pour présenter une expérience poétique des souvenirs. Le jeu comporte quatre scènes (ville enneigée près d'une rivière, vallée ensoleillée au printemps, petit lac dans une forêt pluvieuse, vagues sous une mer agitée) inspirées chacune d'une poésie juéjù. Chaque scène offre un avatar différent, avec un système de contrôle correspondant. Les quatre avatars viennent des peintures «oiseau et fleur ${ }^{35}$ » qui tiennent leur nom du sujet représenté. 
Figure 8. Return: Flower Bird Butterfly and Fish
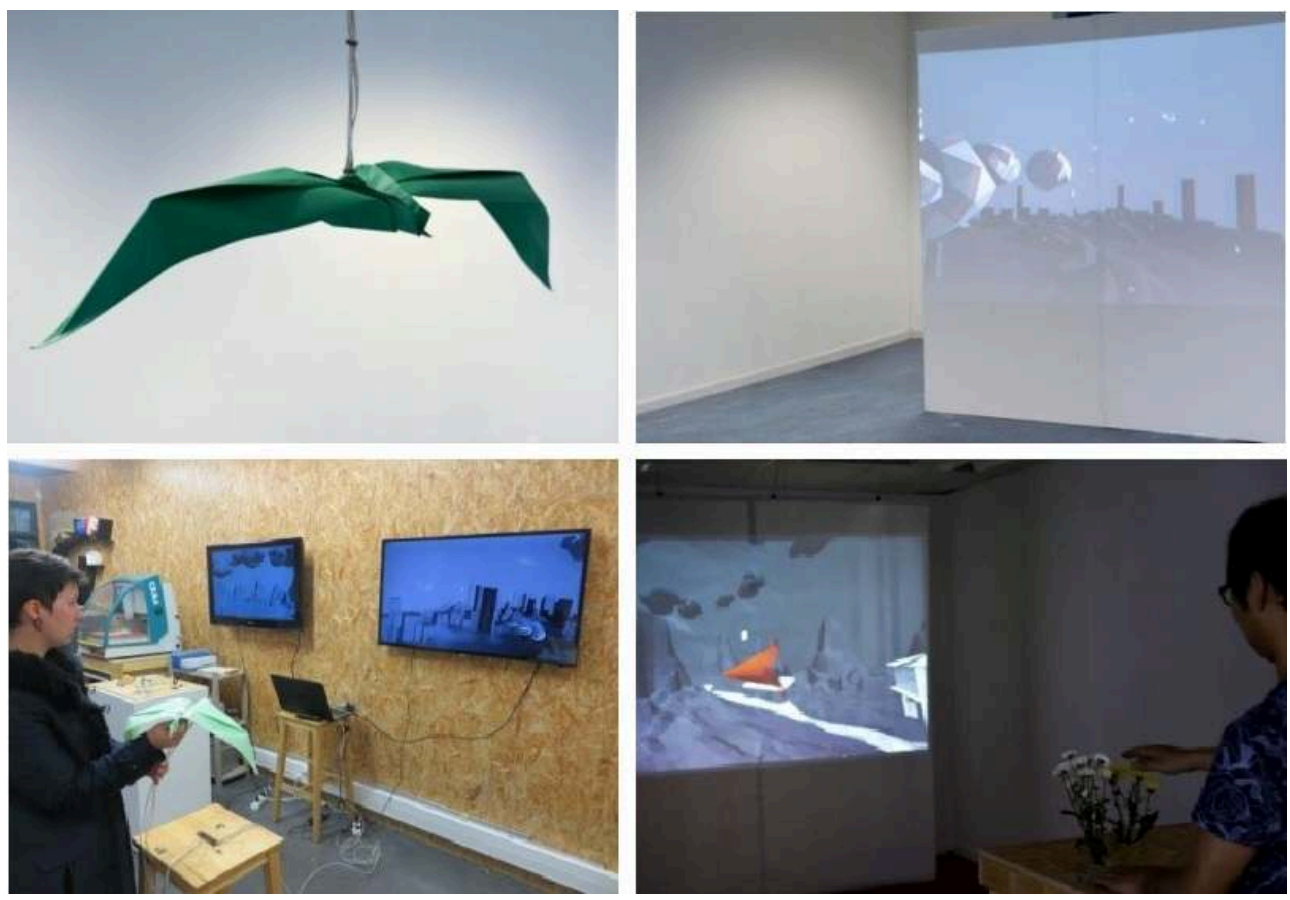

Source: Guofan Xiong, , 2015 (crédits images : Guofan Xiong, 2015)

Le joueur utilisera différentes interfaces pour contrôler chaque avatar afin d'explorer la scène où flottent divers objets, souvenirs d'enfance de personnes issues de différents pays. En circulant autour de l'objet avec un mouvement en spirale correspondant au caractère chinois 回 (retour, rappel...), quatre phrases décrivant la mémoire nous montrent les quatre directions de l'objet. Ensuite, l'objet s'anime. L'environnement en noir et blanc d'origine se colore progressivement. Lorsque tous les souvenirs sont animés, l'espace de jeu devient alors coloré et vivant.

Return se veut un musée de souvenirs permettant de se rappeler et/ou d'échanger avec les nouveaux visiteurs. Dans le jeu, le joueur explore l'espace et rencontre des objets inconnus. Il apprend les histoires de ces derniers par les sons, les images et les textes. Ce jeu vidéo est également une installation permettant de connecter les mouvements d'un corps du monde réel avec ceux du monde virtuel.

Le projet ressemble plus à une expérimentation portant sur la mécanique d'un médium qu'à un jeu cherchant à exprimer quelque chose. Si, dans une certaine mesure, il s'agit d'un projet poétique et personnel, cela ressemble aussi à une collection de formes et de symboles (principalement de juéjù) qui s'articulent autour d'un gameplay simple. Ce projet initia notre intérêt pour la recherche des formes ludiques à partir des formes littéraires (en particulier de la poésie) et nous poussa à créer un jeu plus complexe et significatif dans un cadre de game art.

\section{Traditions et cérémonies à jouer}

Le projet Shangui (山鬼) est un projet qui fonctionne sous un autre angle. L'objectif est de transformer cette littérature ancienne et étrangère en gameplay sans utiliser de représentation textuelle, de présenter sa forme et sa culture comme des expériences à partager avec le public et d'en observer sa compréhension. 

joueurs doivent composer des formes sur la toile avec l'ombre de leur main, pour faire apparaitre des scènes provenant d'un poème traditionnel chinois : les Neuf chants dans le jeu Shangui. Ce gameplay est à la fois une imitation de la cérémonie religieuse du jiŭgē et une représentation physique du caractère ${ }^{37}$. Dans chaque phrase des Chants de $\mathrm{Chu}^{38}$ où il est présent, le caractère 兮 n'a pas de sens réel. Il fonctionne comme un signe du sentiment et donne le rythme de la poésie.

Le chant est musical ou vocal. Ce caractère fonctionne aussi comme structure pour supporter les éléments de symétrie des phrases. Enfin, il agit comme une ponctuation, puisque la ponctuation a été introduite dans la langue chinoise au $\mathrm{xx}^{\mathrm{e}}$ siècle. Nous pouvons dire que 兮 est l'élément le plus important qui définit la forme des Chants de Chu. Dans ce projet d'installation interactive, nous avons décidé de transcrire ce caractère sous la forme de l'action principale physique que les joueurs doivent effectuer (fig. 10).

Figure 9. Installation interactive ludique, Shangui
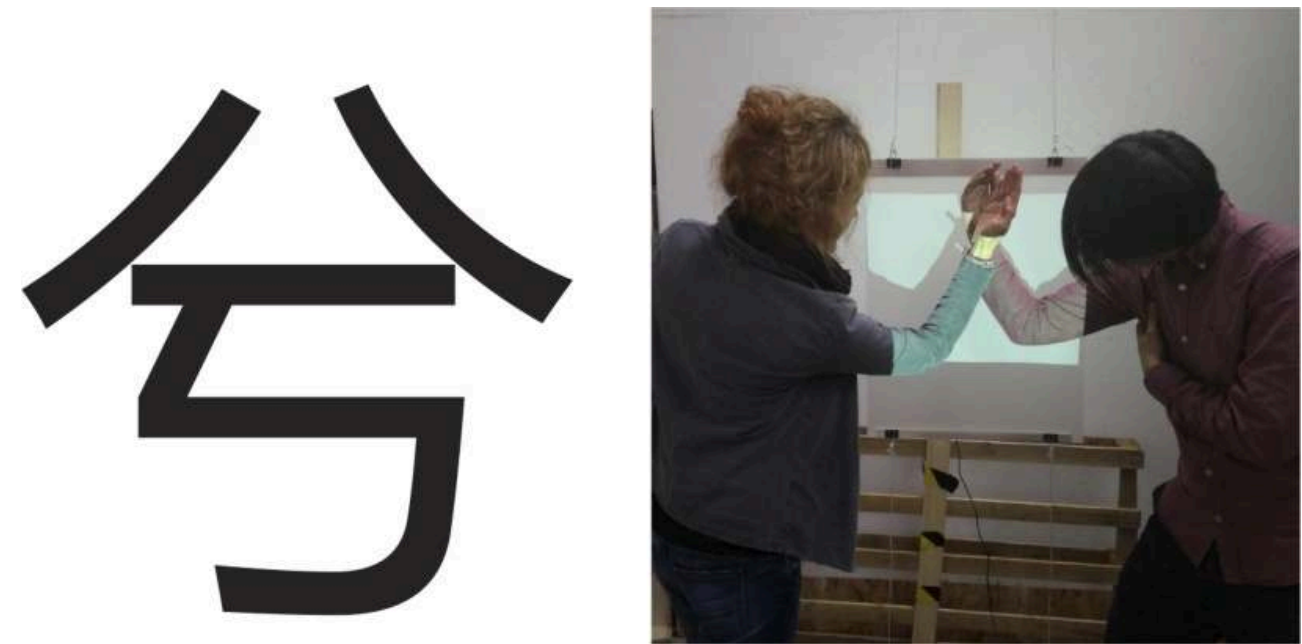

Source : Guofan Xiong, , 2018 (crédits images : Guofan Xiong, 2018)

44

Ce projet est une expérimentation sur la façon dont nous pouvons transformer non seulement les formes à l'intérieur de la poésie, mais aussi les activités culturelles autour de la poésie en gameplay. Notre objectif fut de proposer une installation qui demande aux joueurs de créer leur récit en suivant la méthode du gameplay et donc de favoriser une discussion autour des éléments d'un projet de game art. L'objectif est également de provoquer la construction d'un sens poétique et personnel chez les joueurs et spectateurs.

\section{Conclusion}

Le juéjù est l'une des premières formes de littérature avec lesquelles les enfants chinois entrent en contact. C'est une partie importante de leur première expérience dans l'esthétique et la culture qui l'entoure. Ce style littéraire est simple dans ses formes, à la fois exquis et profond dans son expression. C'est un sujet idéal pour expérimenter ses 
règles dans un projet simple de game art. Il s'agit de créer quelque chose de nouveau dans l'expérience littéraire et l'expérience de jeu.

Le mah-jong est, quant à lui, un jeu de table populaire qui a un gameplay complet. Le lien profond existant entre les règles du mah-jong et du juéjù nous donne un aperçu de la façon dont ces formes et ces règles littéraires peuvent être transformées en gameplay. Il nous montre les raisons pour lesquelles nous ne pouvons pas avoir une expérience poétique en jouant au mah-jong. À l'inverse, le médium jeu vidéo offre des solutions pour présenter des expériences poétiques : liberté du rythme de jeu qui permet aux joueurs de se concentrer sur la découverte de soi et les connexions émotionnelles et système du jeu vidéo qui enrichit l'expérience du jeu et la caractérise avec le style individuel de son auteur.

Bien sûr, la recherche de la poétique dans le jeu vidéo implique de s'affranchir d'une formule industrielle de gameplay standard. Le game art, méthode qui brise les frontières entre les médiums et les formats, pourrait aider à créer un nouvel espace de jeu à la fois poétique et plastique. La recréation d'un gameplay poétique à partir de formes littéraires, qui pourraient être jouées et comprises par des personnes d'une culture différente serait à la fois une extension des formes originales, un hommage et une conservation de la culture elle-même.

\section{BIBLIOGRAPHIE}

Bogost Ian, «A Slow Year : a chapbook of game poems for Atari VCS, PC, and Mac », Ian Bogost. URL : http://bogost.com/games/aslowyear/ [consulté le 01/03/2020]

Bogost Ian, How to do things with videogames, Minneapolis, University of Minnesota Press, coll. « Electronic mediations », 2011.

Cáo Xuĕqín, Le Rêve dans le pavillon rouge [红楼梦, XVIII ${ }^{\mathrm{e}}$ siècle], Pékin, Beijing Publishing House, 2016.

Csíkszentmihályi Mihály, Flow: The Psychology of Optimal Experience, New York, Harper Perennial, 1991.

Csíkszentmihályi Mihály, « Go with the Flow », Wired [en ligne], entretien conduit par John Geirland, 1 septembre 1996. URL : https://www.wired.com/1996/09/czik/ [consulté le 23/06/2020]

Dá Chóngguāng, Huàquán [画筌, 1721], in Chinese Text Project [en ligne]. URL : https://ctext.org/ wiki.pl?if=en\&chapter=326756\&remap=gb [consulté le 05/05/2020]

Demiéville Paul (dir.), Anthologie de la poésie chinoise classique [1962], Paris, Gallimard, coll. « Poésie », 1982.

Ensslin Astrid, Literary Gaming, Cambridge, The MIT Press, 2014.

Goetzmann Marc et Zuppinger Thibaud, « Dossier de l'été $:$ les jeux vidéo, terrain philosophique? », Implications Philosophiques, 2016. URL : http://www.implications- 
philosophiques.org/ethique-et-politique/philosophie-politique/dossier-de-lete-les-jeux-videoterrain-philosophique/ [consulté le 01/03/2020]

Isbister Katherine, How Games Move Us: Emotion by Design, Cambridge, The MIT Press, 2016.

Le Breton Erwan, «Construire des mondes ", Fantasy, retour aux sources, $\mathrm{n}^{\circ} 2$, Éditions multimédias de la BnF, 23 janvier 2020.

Lǐ Qīngzhào, «Shēngshēngmàn » [声声慢, XI ${ }^{\mathrm{e}}-\mathrm{XII}{ }^{\mathrm{e}}$ siècles], in Sòngcísānbăishŏu [東词三百首], Pékin, Zhonghua Book Company, 2009.

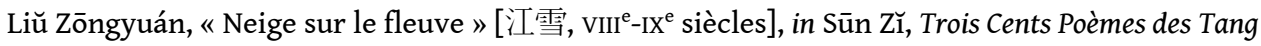
[唐诗三百首, 1763], Pékin, Zhonghua Book Company, 2009.

Maizels Micheal et Jagoda Patrick, The Game Worlds of Jason Rohrer, Cambridge, The MIT Press, 2016.

Montfort Nick, «PVP : Portal versus Passage », Grand Text Auto, 2008. URL : https:// grandtextauto.soe.ucsc.edu/2008/02/24/pvp-portal-versus-passage/ [consulté le 15/01/2018]

Muncy Julie, « Now that I beat The Witness, I see what's wrong with it », Wired [en ligne], 2016. URL : https://www.wired.com/2016/02/the-witness/ [consulté le 15/02/2020]

Schrank Brian, Avant-garde Videogames: Playing with Technoculture, Cambridge, The MIT Press, 2014.

Sjöblom Max et Hamari Juho, « Why do people watch others play video games? An empirical study on the motivations of Twitch users ", Computers in Human Behavior, 2017.

Walters Ashley, « From China to U.S., the game of mahjong shaped modern America, says Stanford scholar ", Stanford News, 2013. URL : https://news.stanford.edu/news/2013/july/ humanities-mahjong-history-071513.html [consulté le 01/05/2020]

Wú Qĭmíng, Shīhuàróngtōnglùn [诗画融通论], Pékin, Zhonghua Book Company, 2018.

Xiong Guofan, Plata Daniel et Chen Chu-Yin, « Huge Balls: an exploration of the comprehension of ludo-narrative elements of a game art installation », EAI Endorsed Transactions on Creative Technologies, $\mathrm{n}^{\circ}$ 21, Gand, European Alliance for Innovation, 2019.

\section{Ludographie}

Blow Jonathan (Thekla Inc.), The Witness, San Francisco, Thekla Inc., 2016, Microsoft Windows. Bogost Ian, A Slow Year, 2010, Microsoft Windows.

Chen Jenova (Thatgamecompany, Santa Monica Studio), Journey, San Mateo, Sony Computer Entertainment, 2012, PlayStation 4.

Chen Jenova (Thatgamecompany), Flower [2009], West Hollywood, Annapurna Interactive, 2017, Microsoft Windows.

Rohrer Jason, Passage, indépendant, 2007, Microsoft Windows.

Vile Monarch, Oh...Sir !! The Insult Simulator, Amsterdam, Good Shepherd Entertainment, 2016, Microsoft Windows.

Viola Bill et Game Innovation Lab, The Night Journey, Los Angeles, USC Games, 2018, Microsoft Windows. 


\section{Installations}

Xiong Guofan, Installation Return: Flower Bird Butterfly and Fish [回花鸟虫鱼], Pau, École

supérieure d'art des Pyrénées, 2015.

Xiong Guofan, Installation Shangui [山鬼], Paris, Exposition Bookniarof, 2018.

\section{NOTES}

1. Selon Marc Goetzmann et Thibaud Zuppinger « le gameplay est l'articulation entre le game, les structures et règles du jeu, et le play, la façon dont le joueur s'approprie les possibilités du jeu en mettant au point ses propres stratégies, pour répondre aux contraintes que les règles "constitutives" du jeu lui imposent ». Marc Goetzmann et Thibaud Zuppinger, « Dossier de l'été : les jeux vidéo, terrain philosophique?», Implications Philosophiques, 2016. URL: http:// www.implications-philosophiques.org/ethique-et-politique/philosophie-politique/dossier-delete-les-jeux-video-terrain-philosophique/ [consulté le 01/03/2020]

2. Le game art implique souvent l'utilisation de jeux vidéo modifiés ou la réutilisation de jeux existants, mais il s'appuie aussi sur un éventail plus large de techniques et de résultats artistiques.

3. Unité phonologique de durée ou de poids d'une syllabe dans certaines langues, une voyelle courte ayant typiquement une more et une voyelle longue deux mores.

4. Nous traduisons : «As games, these rely on the procedural representation of an idea that the player manipulates. As poetry, they rely on the condensation of symbols and concepts rather than the clarification of specific experiences. As images, they offer visually evocative yet obscure depictions of real scenes and objects ». Ian Bogost, «A Slow Year : A chapbook of game poems for Atari VCS, PC, and Mac», Ian Bogost. URL : http://bogost.com/games/aslowyear/ [consulté le 01/03/2020]

5. On nomme ainsi une période d'émergence du jeu vidéo aux États-Unis, entre 1972 et 1983.

6. « Neige sur le fleuve » est un des plus célèbres poèmes de Liŭ Zōngyuán, écrivain de la dynastie Tang né en 773 et mort en 819.

7. Paul Demiéville (dir.), Anthologie de la poésie chinoise classique [1962], Paris, coll. « Poésie », 1982, p. 330-331.

8. Un chow est un groupe de trois tuiles de la même famille (cercles, caractères ou bambous), dont les valeurs se suivent. Un pung est un groupe de trois tuiles rigoureusement identiques, l'équivalent du brelan au poker. Un kong est un groupe de trois tuiles rigoureusement identiques, l'équivalent du carré au poker.

9. "Shēngshēngmàn" est un cí (poème chanté, écrit sur une mélodie préexistante) de Lǐ Qīngzhào, poétesse chinoise de l'époque Song, née en 1084 et morte vers 1151.

10. Nous traduisons.

11. En poésie chinoise, un duilián, ou couplet antithétique, est une paire de lignes poétiques calligraphiées sur une bande de tissu, affichée sur les portes d'entrée des maisons. Le duilián idéal se compose de peu de mots et est porteur d'un sens profond.

12. Le Rêve dans le pavillon rouge, écrit par Cáo Xuĕqín (1723-1763), est un roman de la littérature classique chinoise.

13. Nous traduisons.

14. Nous traduisons.

15. Katherine Isbister, How Games Move Us: Emotion by Design, Cambridge, The MIT Press, 2016, p. 5.

16. Mihály Csíkszentmihályi, Flow: The Psychology of Optimal Experience, New York, Harper Perennial, 1991. 
17. Nous traduisons : «Being completely involved in an activity for its own sake. The ego falls away. Time flies. Every action, movement, and thought follows inevitably from the previous one, like playing jazz. Your whole being is involved, and you're using your skills to the utmost.». Mihály Csíkszentmihályi, "Go with the Flow», Wired [en ligne], entretien conduit par John Geirland, 1 septembre 1996. URL : https://www.wired.com/1996/09/czik/ [consulté le 02/03/2020]

18. Katherine Isbister, op. cit., p. 5-6.

19. Ibid., p. 15-17.

20. Wú Qǔmíng, Shīhuàróngtōnglùn, Pékin, Zhonghua Book Company, 2018.

21. Pêcheur à la ligne solitaire au bord d'une rivière en hiver de Mă Yuăn et de Zhū Ruì au musée national de Tōkyō, Japon. URL: http://www.emuseum.jp/result? mode=simple\&d_lang=fr\&s_lang=fr\&word=P\%C3\%AAcheur+

$\%$ C3\%A0+la+ligne+solitaire+au+bord+d\%E2\%80\%99une+rivi\%C3\%A8re

22. Mă Yuăn, est un peintre chinois né vers 1160 et mort vers 1225 .

23. Zhū Ruì, est un peintre impérial de la dynastie Ming, période Zhengde (1506-1521).

24. Dá Chóngguāng, est un homme politique, peintre et calligraphe de la dynastie Qing, né en 1623 et mort en 1692 .

25. Nous traduisons : 《虚实相生, 无画处皆成妙境 》. Dá Chóngguāng, Huàquán [1721], Chinese Text Project. URL : https://ctext.org/wiki.pl?if=en\&chapter=326756\&remap=gb [consulté le 05/05/2020]

26. Ian Bogost, How to do things with videogames, Minneapolis, University of Minnesota Press, coll. « Electronic mediations », 2011.

27. Brian Schrank, Avant-garde Videogames: Playing with Technoculture, Cambridge, The MIT Press, 2014, p. 151-152.

28. Nick Monfort, «PVP: Portal versus Passage », Grand Text Auto, 2008. URL: https:// grandtextauto.soe.ucsc.edu/2008/02/24/pvp-portal-versus-passage/ [consulté le 15/01/2018]

29. Nous traduisons : "Now that you've been reminded of your own mortality, what are you going to do with the rest of your life?»

30. Micheal Maizels et Patrick Jagoda, The Game Worlds of Jason Rohrer, Cambridge, The MIT Press, 2016, p. 26.

31. Max Sjöblom, et Juho Hamari, « Why do people watch others play video games? An empirical study on the motivations of Twitch users ", Computers in Human Behavior, 2017, p. 2.

32. Erwan Le Breton, "Construire des mondes», Fantasy, retour aux sources, $\mathrm{n}^{\circ} 2$, Éditions multimédias de la BnF, 23 janvier 2020.

33. Julie Muncy, « Now that I beat The Witness, I see what's wrong with it », Wired [en ligne], 2016. URL : https://www.wired.com/2016/02/the-witness/ [consulté le 15/02/2020]

34. Guofan Xiong, Daniel Plata et Chu-Yin Chen, «Huge Balls: An exploration of the comprehension of ludo-narrative elements of a game art installation ", EAI Endorsed Transactions on Creative Technologies, $\mathrm{n}^{\circ}$ 21, Gand, European Alliance for Innovation, 2019.

35. École artistique de la peinture chinoise qui présente une esthétique poétique et un sentiment doux et distingué.

36. Les Neuf chants sont des poèmes attribués à Qū Yuán, qui a vécu aux alentours de $300 \mathrm{av}$. J.-C.

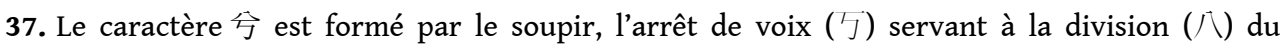
discours (musique, poésie, phrases) : sorte de césure ou d'interponction phonique.

38. Les Chants de Chu sont une anthologie de dix-sept poèmes. Ils sont originaires du royaume de Chu et datent de l'époque des Royaumes combattants, $\mathrm{IV}^{\mathrm{e}}$ et $\mathrm{III}^{\mathrm{e}}$ siècles av. J.-C. 


\section{RÉSUMÉS}

Comment pouvons-nous recréer des jeux originaux inspirés des formes poétiques littéraires, étroitement liées à leur contexte culturel, et offrant aux joueurs des expériences ludiques et poétiques? Dans cet article, nous analyserons les liens profonds entre le jeu de table traditionnel mah-jong et la forme poétique chinoise juéjù, afin de déterminer comment ces formes poétiques sont représentées par les règles du jeu et d'autres éléments ludiques. En les comparant avec des exemples de jeux vidéo traitant d'un sujet similaire, nous verrons quelles inspirations peuvent contribuer à créer un gameplay support d'expérience poétique, qui présente à la fois les formes et le sens de la poésie. Par l'exploration de ces formes dans des projets de game art, nous allons découvrir l'espace poétique potentiel de médium du jeu vidéo.

\section{INDEX}

Mots-clés : game art, gameplay, médium artistique et culturel, art numérique, poésie, formes littéraires, rhetoric procedural

\section{AUTEURS}

\section{GUOFAN XIONG}

Doctorant sous la direction de Chu-Yin Chen et la co-direction de Sophie Daste, Image numérique et réalité virtuelle (Inrev) du Laboratoire Arts des images et art contemporain (Aiac, EA-4010) de l'École doctorale esthétique, sciences et technologies des arts (Edesta), Université de Paris, bâtiment D - $3^{\mathrm{e}}$ étage - salle D302, 2 rue de la liberté 93526 Saint-Denis cedex. Étudiantchercheur, groupe Spatial média de l'Ensadlab (laboratoire de recherche en art et en design de l'École nationale supérieure des arts décoratifs), 31 rue d'Ulm 75240 Paris cedex 05 ; guofan.xiong@ensad.fr

\section{CHU-YIN CHEN}

Professeur, Image numérique et réalité virtuelle (Inrev) du laboratoire Arts des images et art contemporain (Aiac, EA-4010) de l'École doctorale esthétique, sciences et technologies des arts (Edesta), Université de Paris, bâtiment D - $3^{\mathrm{e}}$ étage - salle D302, 2 rue de la liberté 93526 SaintDenis cedex ; chu-yin.chen@univ-paris8.fr 